\title{
SOFTWARE FOR MODELING, ANALYSIS AND DESIGN OF FILTERS WITH LC SECTIONS AND QUARTZ CRYSTALS
}

\author{
Miroslav Lutovac ${ }^{1}$, \\ Vladimir Mladenović
}

'Singidunum University, 32 Danijelova Street, Belgrade, Serbia

${ }^{2}$ University of Kragujevac, Faculty of Technical Sciences, Čačak, Serbia

Correspondence:

Miroslav Lutovac

e-mail:

mlutovac@singidunum.ac.rs

\begin{abstract}
:
Filters that consist of LC sections and quartz crystals can be modelled by LC equivalent sections and represented as rational functions of very high order. In this paper, we present software that can be used as the template notebook for modeling, analysis and design of the known filter structure. A method for automated generation of schematic model of crystal filter as a ladder LC structure is described. The filter transfer function can be derived as closed-form expression from schematic model so that other analyses can be performed, such as sensitivity analysis and parameter optimization in order to satisfy the desired filter specifications.
\end{abstract}

Key words:

crystal filters, LC filter, quartz filter.

\section{Acknowledgment:}

This work was supported by the Ministry of Education, Science and Technological Development of the Republic of Serbia under Grant TR 32048.

\section{INTRODUCTION}

The analysis and design of lossless LC filters are extensively covered in numerous books and papers (Lutovac et al., 2015). That was the main reason to use the established methodology and developed procedures to design other types of filters. Manual derivation of transfer functions is possible only for low-order circuits.

The design and analysis of filters with small number of elements can also be time-consuming in the case when each element is replaced with a more complex circuit model. Although numeric software can be used for completing the most frequently used task, the symbolic approach shows benefits in optimization.

Application of conventional software for the design and analysis of complex filter structure is subject to errors due to the limited accuracy of numerical methods. The analysis of the impact on the value of certain parameters can be done by inverse engineering, but they often make mistakes due to the limited accuracy. In the existing software solutions, only approximate and empirical methods were used.

The problem can be solved exactly using computer algebraic methods so that all filter parameters can be described using symbols (Wolfram, 
2015). Symbols can be substituted by numeric values only when it is necessary, for example, for plotting the graphics of some filter functions. When the complexity of the functions is extremely large, certain parameters can be represented as a ratio of integers, and thus retain the accuracy of the final results.

The fact that all parameters are symbols (including complex frequency) provides the possibility to derive the frequency of extremes, to approximate function in a certain range of interest, to replace each element with the more complex electrical model, or to compute the sensitivity function to element values. In this way, the analysis of the impact of certain parameters is facilitated and optimal value of some parameters can be computed under some constrains. As an example, the resonant frequency of a complex filter can be expressed in terms of some filter elements.

\section{NEW SOFTWARE - TECHNICAL SOLUTION}

The technical solution - new software (released in 2015) is tested at the Institute "Mihajlo Pupin" in Belgrade as a scientific result of the project TR32048. The authors of this software are Miroslav Lutovac, Snežana Dedić - Nešić, Irini Reljin, Ana Gavrovska, and Dubravka Jevtić. This paper presents the main principles that were used for developing this software.

Software is distributed in the form of notebook, with all descriptions and program. The first part of the notebook consists of interactive drawing schemes where crystal units are described as impedance. All the essential characteristics can be automatically derived from the scheme. Verification was done using numeric software.

\section{Notebook Format}

The main part of the program looks like a separate file formatted as a chapter in the book, with the title, the subtitle and text descriptions, as shown in Figure 1.

\section{Software for modeling, analysis, and design of quartz crystals filters using computer algebra system}

Miroslav Lutovac, Snežana Dedić - Nešić,

Irini Reljin, Ana Gavrovska, Dubravka Jevtić

New software - technical solution M85, 2015

SetDirectory [NotebookDirectory []]

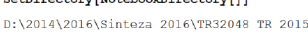

Figure 1. The first part of the notebook with title and subtitle.

\section{Loading Knowledge}

The special folder contains the files with the knowledge that is derived and tested in another project, also available as the technical solution for the project TR32048 (developed in 2014).

Figure 2 presents part of knowledge that contains the model of quartz crystal unit as impedance, real part and the imaginary part.

Figure 3 shows part of the program that loads knowledge from several files. The purpose of one knowledge file is to clear predefined variable definitions so that new variables can be used without interfering with previous program executions. The last file contains the pure functions that will be used for computations in this main program.

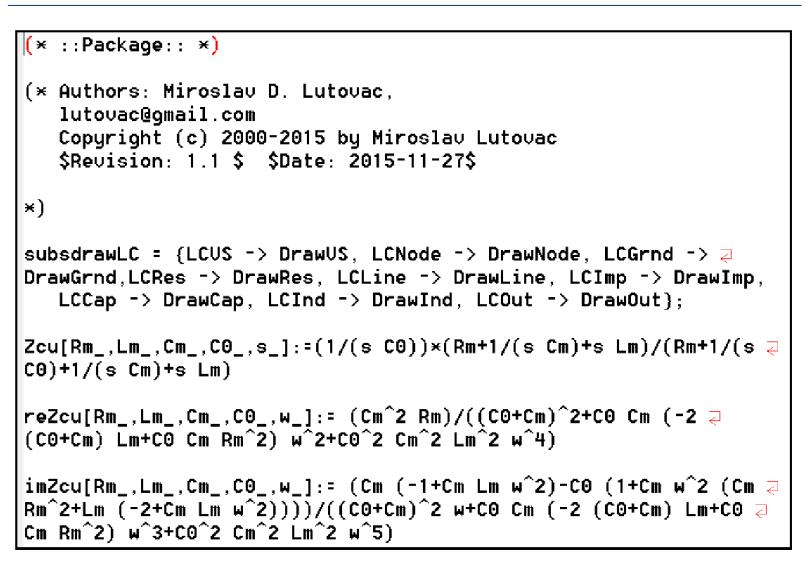

Figure 2. Knowledge thas is saved in separate file and loaded in the notebook.

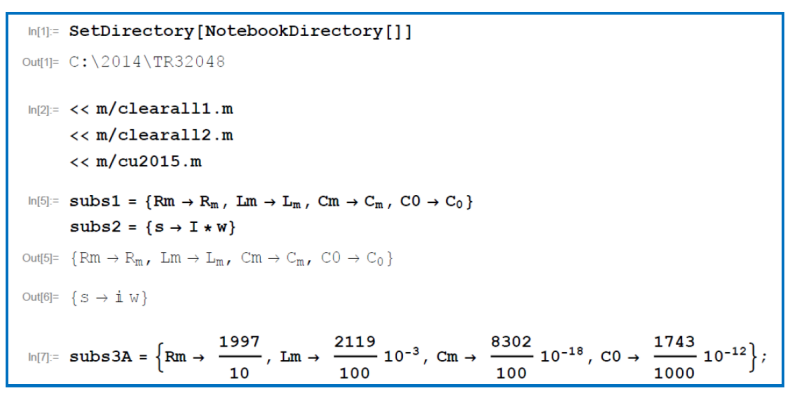

Figure 3. Loading knowledge in the main program.

\section{Verification of Knowledge and Characteristics}

After loading the knowledge from the third file cu2015.m, the values of elements can be represented as ratio of integers, and thus all further derivations are exact. 
Figure 4 illustrates that the pure function entered into the main program (that contains previously saved knowledge of the imaginary part of the impedance of the crystal unit) can be used in the same manner as built-in function.

This approach is particularly useful when the derived functions are already verified, and it is not necessary to re-check the accuracy of the formula. Figure 4 is used in this program just to perform the comparison with the previously published results and to show that the function curve is correct with identical numerical values of parameters.

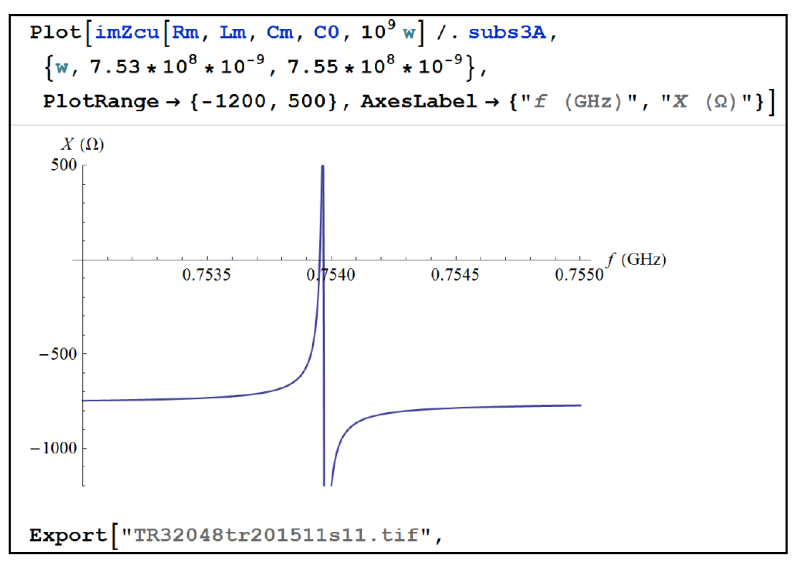

Figure 4. Drawing characteristics of function loaded into the main program.

\section{Using Knowledge for Deriving new Properties}

The last line of the code in Fig. 4 shows how the resulting graphics can be exported in the corresponding figure format with the required resolution.

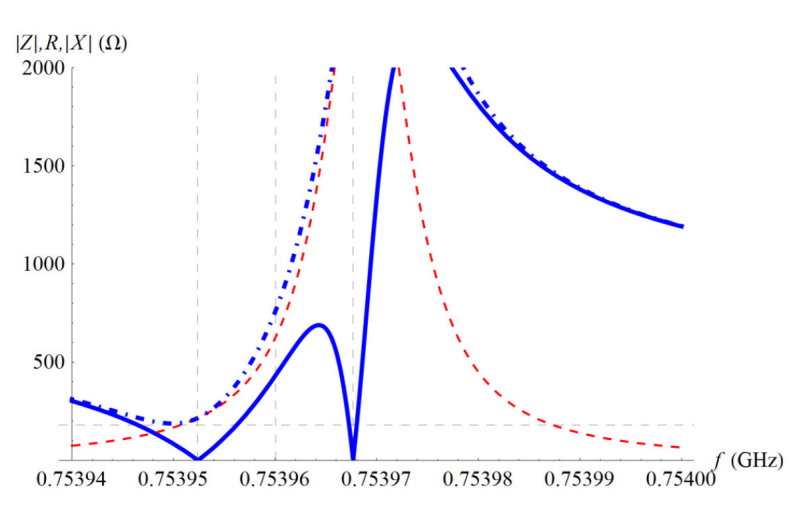

Figure 5. Illustrating more characteristics of the crystal unit.
Figure 5 presents the impedance, real and imaginary part of impedance of the crystal unit, where all main features can be observed, such as the frequency when the imaginary part is equal to zero or where there is the minimum of the impedance.

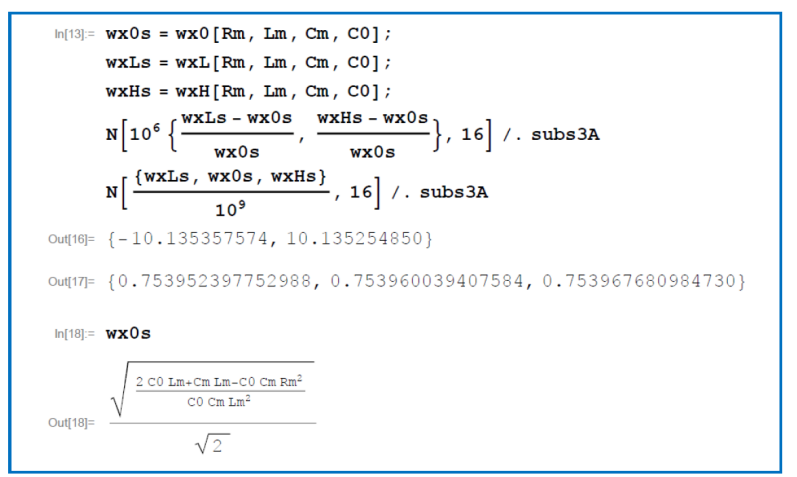

Figure 6. Calculation of the characteristic frequency of the crystal unit.

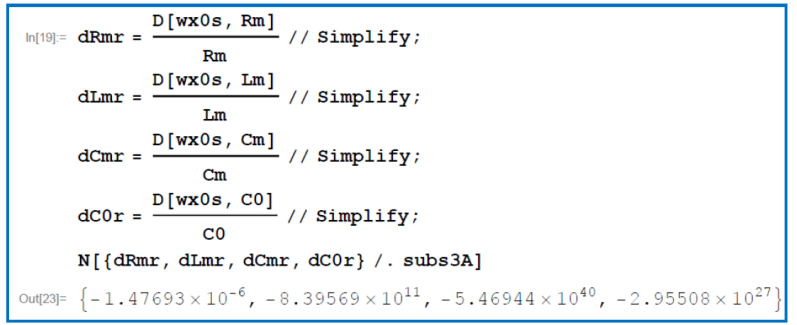

Figure 7. Calculation of the sensitivity of the crystal unit.

Figure 6 shows how the pure functions that are loaded as knowledge can be used for deriving other function in the symbolic form, and how to compute for specific numeric parameters. Each function can be called with the symbolic values of the arguments, which makes it possible to calculate some other functions such as frequency sensitivity to changes in the parameters of the crystal units, as illustrated in Figure 7.

When numerical values have to be computed, a substitution rule is performed using notation "/.".

\section{EMBEDDING KNOWLEDGE INTO SYSTEM}

The second part of the software uses the knowledge of the crystal units for the design of more complex structures. For example, a filter which consists of 6 quartz crystal units, such that the two individual crystals are of type ZA, two of type IA, and one type $\mathrm{ZB}$ and $\mathrm{ZC}$ 
(different types means the same structure but different element values). Other components of the filter as resistors, capacitors, and coils, are marked in the figures with symbolic values.

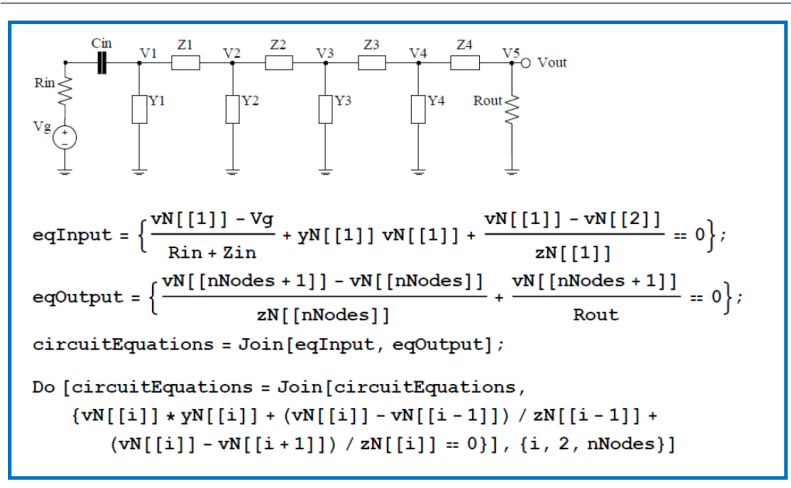

Figure 8. Automated drawing and setting up a system of equations.

The basic concept of this software solution is to define the system structure, in this case a ladder structure, and to automate the design procedure. The more general structure can be modified using substitution rules, where each element of the ladder structure is replaced with more complex element models. The initial structure can be used for setting the system of equations or drawing the system, so that technical documentation is prepared during the design stage. Knowledge of some elements (such as mathematical and electrical model of crystal units) is tested in the first part of the software description. This way, we avoid typesetting errors because electrical schematic and mathematical representations are the result of the same initial system structure.

For systems that have a repeated structure, the list of specification for schematic drawings and description of the system by equations are done using the simple program such as that in Figure 8.

Knowledge with drawing functions can also be saved in a separate file and loaded into the main program before drawing the system. For drawing the system, different symbolic names can be used. The names of the variables can be prepared as a separate substitution list, and the same replacement rules can be used for drawing and solving the system. As solving relies on symbolic manipulation, substitution of the simple model with the more complex model is performed at the final stage.

After solving the equations, the obtained result of the transfer function can be so complex that it cannot be displayed, as seen in Figure 9. More than 5500 symbols are omitted in the reported result. This example shows that it is impractical manual solving of the system.

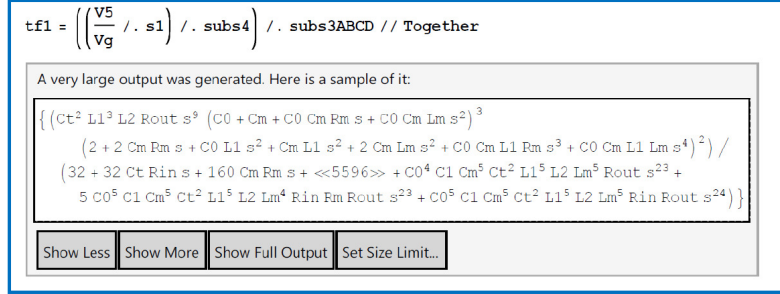

Figure 9. Symbolic result that cannot be displayed.

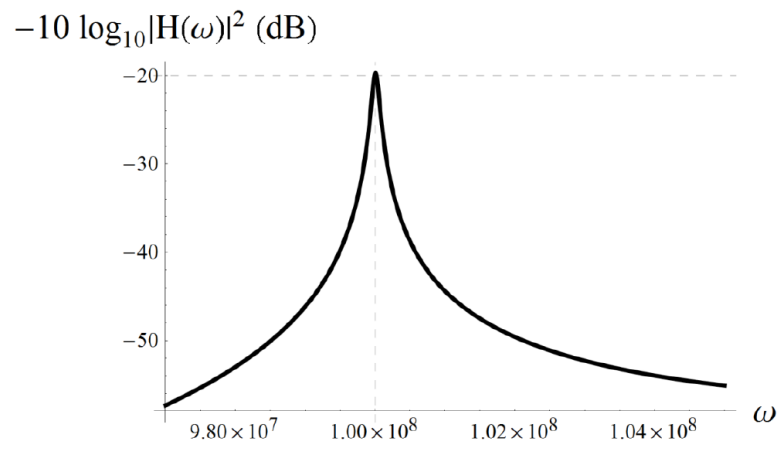

Figure 10. Filter attenuation of the crystal filter.

In a similar manner, the magnitude function and the filter attenuation can be derived, as shown in Figure 10.

Although expressions are very complex, they may be used for further derivations, such as the sensitivity of the magnitude function with respect to any filter parameters. Graphics of sensitivity with respect to three elements of the filter are shown in Figures 11, 12, and 13. It is important to notice that all three sensitivities are equal to zero at the resonant frequency. This means that the low-sensitivity properties of lossless ladder structures are retained in the more complex system when passive components are replaced by crystal units.

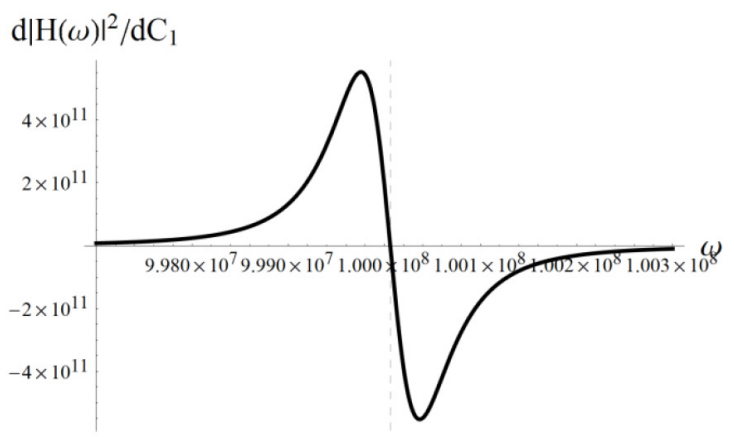

Figure 11. The derived sensitivity with respect to $C_{1}$. 


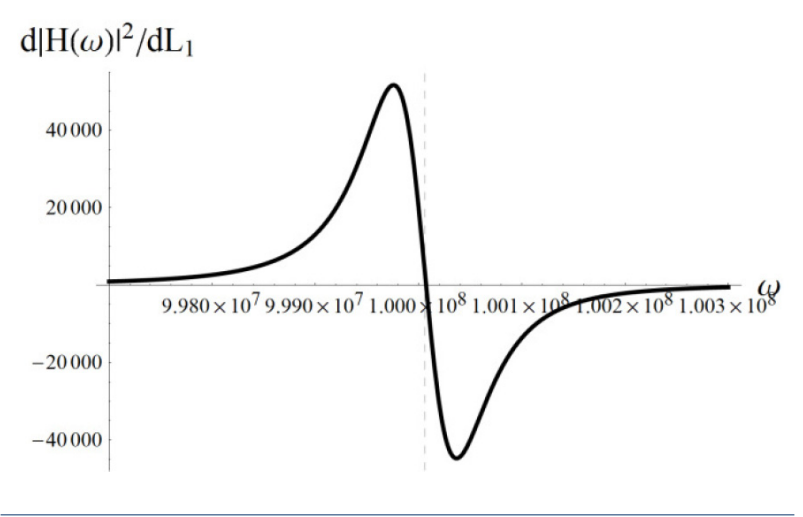

Figure 12. The derived sensitivity with respect to $L_{1}$.

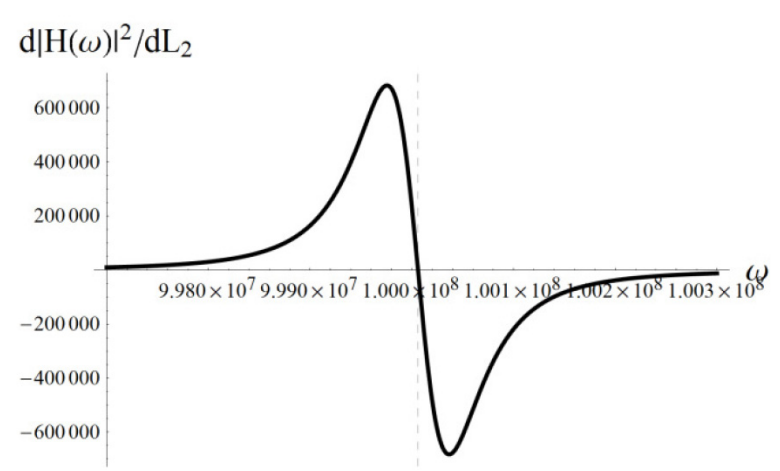

Figure 13. The derived sensitivity with respect to $C_{2}$.

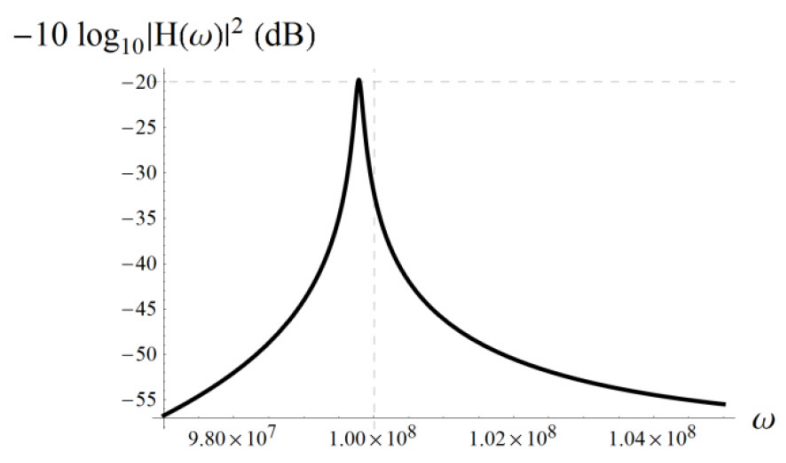

Figure 14. Magnitude response for increased value $C_{1}$ of $1.5 \%$.

The robustness of the magnitude function with respect to element tolerances can be proved by drawing magnitude response for different element values, as illustrated in Figures 14, 15, and 16. This result is important for fabrication of crystal filter, because it provides the influence of each element and how to organize testing and tuning strategy.

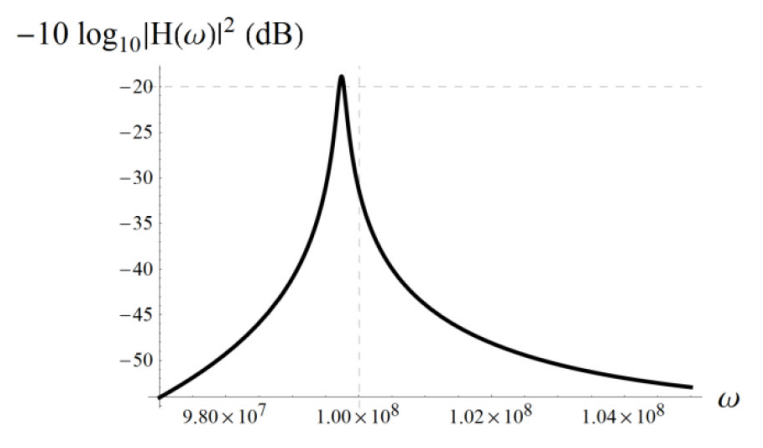

Figure 15. Magnitude response for increased value $L_{1}$ of $8 \%$.

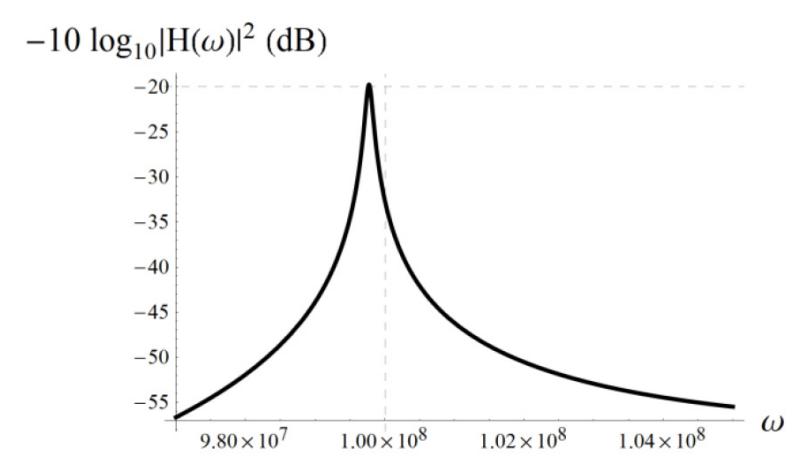

Figure 16. Magnitude response for increased value $C_{2}$ of $0.5 \%$.

\section{SUMMARY}

The software described in this paper presents the referenced document that contains all required knowledge and procedures for modeling, analysis and design of quartz crystals filters. After entering only a number of filter sections, the rest of the procedure is automated. The future work is to provide graphical user interface, while the knowledge from this software can be saved as a separate file.

\section{REFERENCES}

Dujković, D., Dedić Nešić, S., Grubišić, Ana Gavrovska, A., \& Reljin, I. (2013). High-quality Crystal Filter - Design and Realization. TELFOR Journal, 5(2), 118-122.

Paskaš, M., Lutovac, M., Dujković, D., Reljin, I., \& Reljin, B. (2012). Computer Model for Analysis and Re-design of Crystal Filters. In Proc. Small Systems Simulation Symposium 2012, 12-14 February 2012, (pp. 106-110). Niš, Serbia: Yugoslav Simulation Society.

Lutovac, D., Tosic, V. \& Evans L. (2001). Filter Design for Signal Processing Using MATLAB and Mathematica. Upper Saddle River, Prentice Hall.

Wolfram, S. (2015). An Elementary Introduction to the Wolfram Language. Champaign, IL: Wolfram Media. 